\title{
CD-PLLD co-delivering docetaxel and MMP-9 siRNA plasmid for nasopharyngeal carcinoma therapy in vivo
}

\author{
TAO LIU ${ }^{1}$, XIDONG WU ${ }^{2}$, YIGANG WANG ${ }^{3}$, XIONGJUN HOU ${ }^{4}$, \\ GANG JIANG $^{1}$, TING WU ${ }^{5}$, HUIFEN XIE ${ }^{1}$ and MINQIANG XIE ${ }^{1}$ \\ ${ }^{1}$ Department of Otolaryngology, Zhujiang Hospital, Southern Medical University, Guangzhou, \\ Guangdong 510282; ${ }^{2}$ Department of Pharmacology, Jiangxi Institute of Materia Medica, Nanchang, \\ Jiangxi 330029; ${ }^{3}$ School of Life Sciences, Zhejiang Sci-Tech University, Hangzhou, Zhejiang 310018; \\ ${ }^{4}$ Department of Pharmacy, Jiangxi Provincial People's Hospital, Nanchang, Jiangxi 330006; \\ ${ }^{5}$ Department of Light Chemical Engineering, Guangdong Polytechnic, Foshan, Guangdong 528041, P.R. China
}

Received November 5, 2015; Accepted November 29, 2016

DOI: $10.3892 / \mathrm{mmr} .2017 .6715$

\begin{abstract}
The co-delivery of a drug and a target gene has become a primary strategy in cancer therapy. Based on our previous study, a synthesized star-shaped co-polymer consisting of $\beta$-cyclodextrin (CD) and a poly(L-lysine) dendron (PLLD) was used to co-deliver docetaxel (DOC) and matrix metalloproteinase 9 (MMP-9) small interfering RNA, via CD-PLLD/DOC/MMP-9 complexes, into mice implanted with HNE-1 human nasopharyngeal carcinoma (NPC) tumor cells in vivo. Unlike the commonly used amphiphilic co-polymer micelles, the obtained $\mathrm{CD}$ derivative may be used directly for a combined delivery of nucleic acid and hydrophobic DOC without a complicated micellization process. In vivo assays demonstrated that CD-PLLD/DOC/MMP-9 inhibited HNE-1 tumor growth and decreased proliferating cell nuclear antigen expression levels, indicating a potential strategy for NPC therapy. In addition, the distribution of DOC and MMP-9 was investigated; CD-PLLD/DOC/MMP-9 complexes were phagocytized in reticuloendothelial systems, including the liver and spleen, which requires further study. Furthermore, the complexes did not cross the blood-brain barrier due to their large molecular size, suggesting they may be relatively safe. Additionally, the complexes mediated increased DOC concentrations with prolonged blood circulation and EGFP expression in HNE-1 tumors. These results suggest the future potential application of CD-PLLD/DOC/MMP-9 for NPC therapy.
\end{abstract}

Correspondence to: Dr Tao Liu or Professor Minqiang Xie, Department of Otolaryngology, Zhujiang Hospital, Southern Medical University, 253 Industrial Avenue, Guangzhou, Guangdong 510282, P.R. China

E-mail: taoliu18@126.com

E-mail: min_qiang_x@hotmail.com

Key words: star-shaped, poly(L-lysine) dendrimer, co-delivery, nasopharyngeal carcinoma

\section{Introduction}

Nasopharyngeal carcinoma (NPC) is one of the most common types of malignant tumor in southern China (1). Currently, a combination of radiotherapy and chemotherapy is the primary clinical treatment for NPC. However, the average five-year survival rate of patients with advanced NPC remains low due to tumor metastasis and multi-drug resistance (2).

A recent study demonstrated that combining chemotherapy with gene therapy may mediate synergistic actions, enhance treatment effects and inhibit the development of drug resistance (3). Furthermore, He et al (4) developed a drug and gene co-delivery system, using zinc ions $\left(\mathrm{Zn}^{2+}\right)$ as the connecting point. The obtained coordination liposome mediated the cellular uptake of cisplatin and small interfering (si)RNA, and enabled efficient endosomal escape in cisplatin-resistant ovarian cancer cells. Li et al (5) used poly (carboxybetaine) to conjugate camptothecin, and assembled cationic liposomes to form a drug and gene dual-carrier, which demonstrated a synergistic tumor suppression effect in tumor-bearing mice in vivo. Chang et al (6) assembled drug and gene co-delivering liposomes from amphiphilic pillar[5]arene capped with ferrocenium, which exhibited redox sensitivity and effective drug/siRNA co-delivery.

Previous studies have incorporated hydrophobic anticancer drugs into the hydrophobic cores of these carriers, and bound plasmid DNA or siRNA to cationic hydrophilic shells. However, the self-assembly processes involved in the preparation of micelles are typically difficult to control, and therefore stable and uniform complexes are difficult to obtain. Furthermore, micelles are not stable in blood circulation in vivo, and their disassembly may result in drug emission (7-9). A recent study synthesized a star-shaped cyclodextrin (CD) derivative, consisting of a $\mathrm{CD}$ core and poly(L-lysine) dendron (PLLD) arms (CD-PLLD) (10). The CD core may interact with hydrophobic drug models, thus avoiding the complicated micellization process.

For gene delivery, hyperbranched or dendrimer cationic polymers, including poly (amidoamine)s, have been widely used and have demonstrated efficiency due to their topologic 
structure $(11,12)$. Of these, PLLD and its derivatives are of interest due to their well-defined architecture, good biocompatibility, low cytotoxicity, high transfection efficiency and biodegradability. Previous studies have investigated their synthesis and potential applications in drug or gene delivery (13-15). The synthesized CD-PLLD demonstrated stability and good gene delivery ability in our previous study (10), and was used to co-deliver docetaxel (DOC) and a matrix metalloproteinase 9 (MMP-9) siRNA plasmid for NPC therapy (16). The present study investigated the distributions of MMP-9 and DOC in vivo, and the potential of using the CD-PLLD/DOC/MMP-9 complex for NPC treatment. In addition, it was demonstrated in vitro that CD-PLLD may co-deliver DOC and MMP-9 effectively into HNE-1 NPC cells, and that these cells underwent apoptosis, therefore suggesting its potential application in drug and gene co-delivery.

\section{Materials and methods}

Materials. CD-PLLD/DOC/MMP-9 complexes were synthesized according to our previous study (the loading amount of DOC was $13.20 \mu \mathrm{g} / \mathrm{mg}$ ) (16). DOC was purchased from Sigma-Aldrich; Merck Millipore (Darmstadt, Germany) and used without further purification. The most efficient interference MMP-9 siRNA plasmid vector expressing enhanced green fluorescent protein (EGFP), selected following screening (16), was purchased from Invitrogen; Thermo Fisher Scientific, Inc. (Waltham, MA, USA). The optimum siRNA sequences targeting MMP-9 were as follows: Forward, 5'-TGC TGAAACCGAGTTGGAACCACGACGTTTTGGCCACTG ACTGACGTCGTGGTCAACTCGGTTT-3' and reverse, 5'-CCTGAAACCGAGTTGACCACGACGTCAGTCAGTGG CCAAAACGTCGTGGTTCCAACTCGGTTTC-3'. Tert-butyl methyl ether and acetonitrile were of analytical grade and used without further purification. HNE-1 human NPC cells were provided by Southern Medical University (Guangzhou, China). A total of 50 female specific pathogen-free BALB/c nude mice (age, 4-5 weeks; weight, 17-20 g) maintained at a specific pathogen free facility with a constant humidity (60\%) and temperature $\left(28^{\circ} \mathrm{C}\right)$ at $12 / 12 \mathrm{~h} \mathrm{light/dark}$ cycle with free access to food and water were obtained from the Center for Laboratory Animal Sciences, Southern Medical University. Ethical approval was obtained from The Institutional Administration Panel for Laboratory Animal Care of Southern Medical University. The university guidelines for care and use of laboratory animals were strictly followed.

In vivo metabolism assay in nude mice. Nude mice implanted with HNE-1 tumors were used as the animal model for in vivo analyses. HNE-1 cells ( $1 \times 10^{7}$ cells in $200 \mu \mathrm{l}$ PBS) were injected subcutaneously into the right axillary flank of female BALB/c nude mice. Once the tumors had grown to a maximum of $1 \mathrm{~cm}$ in diameter, the mice were sacrificed by cervical dislocation, and the HNE-1 tumors were removed and sectioned into $2 \times 2 \times 2 \mathrm{~mm}$ small pieces in aseptic conditions. The tumors were subsequently transplanted into the right axillary flanks of five mice. Following this, all mice were anesthetized by an intraperitoneal injection of $0.1 \mathrm{ml}$ chloral hydrate (Shanghai Aladdin Bio-Chem Technology Co., Ltd., China), and injected with CD-PLLD containing $20 \mu \mathrm{g} / \mathrm{g}$ DOC and $6 \mu \mathrm{g} / \mathrm{g}$ MMP-9 via the tail vein. Mice were sacrificed by cervical dislocation 5 , 30 or 60 min later and organs were harvested for high-performance liquid chromatography (HPLC), or $48 \mathrm{~h}$ later for EGFP detection.

Standard curves of DOC concentration for in vivo distribution. Standard curves of DOC concentration in each tissue (liver, kidney, lung, heart, spleen, brain and HNE-1 tumor) were obtained by HPLC analysis. Briefly, $12.16 \mathrm{mg}$ DOC standard samples were transferred into a $5 \mathrm{ml}$ calibrated flask. The DOC solution was subsequently diluted with mobile phase solvent consisting of methanol and purity water $(70 / 30, \mathrm{v} / \mathrm{v})$ to achieve the following gradient DOC concentrations: 1,216, $608,304,152$ and $76 \mu \mathrm{g} / \mathrm{ml}$. Tissue samples harvested from nude mice bearing HNE-1 tumors were rinsed with ice-cold saline, dried on filter paper, weighed and homogenized with saline ( $\mathrm{g}: 2 \mathrm{ml}$ ratio of tissue weight:saline dosage). Following this, $160 \mu \mathrm{l}$ tissue sample and $40 \mu \mathrm{l}$ prepared DOC standards sample solution with gradient concentration were mixed, and $1 \mathrm{ml}$ tert-butyl methyl ether was added into each sample and mixed for $5 \mathrm{~min}$ by vortex for extraction. The total organic layer was separated by centrifugation at $11,180 \mathrm{x} \mathrm{g}$ for $10 \mathrm{~min}$ at $4^{\circ} \mathrm{C}$, transferred to a clean tube and evaporated to dryness at $40^{\circ} \mathrm{C}$ under a stream of nitrogen. The drug residue was finally reconstituted in $0.4 \mathrm{ml}$ acetonitrile followed by centrifugation at $1,1180 \mathrm{x} \mathrm{g}$ for $5 \mathrm{~min}$ at $4^{\circ} \mathrm{C}$ prior to analysis, of which $10 \mu l$ supernatant fluid was injected into the HPLC system. The HPLC analysis of DOC was achieved on a $\mathrm{C}_{18}$ column (Waters Corporation, Milford, MA, USA) with a mobile phase consisting of methanol and purity water (70/30, v/v) at a flow rate of $1.0 \mathrm{ml} / \mathrm{min}$. The effluents were monitored at $227 \mathrm{~nm}$ and quantized by comparing the peak areas with the standard curve.

DOC distribution in vivo. Following DOC administration, nude mice bearing HNE-1 tumors were sacrificed after 5, 30 or $60 \mathrm{~min}$, and the tumors and aforementioned tissues were rapidly dissected. Samples were rinsed with ice-cold saline, dried on filter paper and homogenized with saline at a 1:2 ratio of tumor weight: Saline dosage. Following this, $0.2 \mathrm{ml}$ of tissue sample and $1.2 \mathrm{ml}$ tert-butyl methyl ether were mixed for $5 \mathrm{~min}$ by vortex to extract, and the total organic layer was separated by centrifugation at $11,180 \mathrm{x}$ g for $10 \mathrm{~min}$ at $4^{\circ} \mathrm{C}$, transferred to a clean tube, and evaporated to dryness at $40^{\circ} \mathrm{C}$ under a stream of nitrogen. The drug residue was subsequently reconstituted in $0.4 \mathrm{ml}$ acetonitrile and centrifuged at $11,180 \mathrm{x} \mathrm{g}$ for $5 \mathrm{~min}$ at $4^{\circ} \mathrm{C}$ prior to analysis. The DOC concentrations were determined by HPLC analysis, which was performed using the aforementioned method.

EGFP expression in vivo. Nude mice were sacrificed by cervical dislocation after $48 \mathrm{~h}$ via tail vein injection of nanocomposites. HNE-1 tumor and the aforementioned tissues were immediately dissected, and EGFP expression levels in frozen sections of tumor tissue were observed via fluorescence microscope.

In vivo tumor inhibition. Nude mice implanted with HNE-1 tumors were used as the animal model to assess the efficacy of CD-PLLD/DOC/MMP-9 against tumor cells in vivo. The mice 
were randomly divided into four groups $(n=5)$. Subsequently, the mice were intravenously injected via the tail vein with $250 \mu \mathrm{l}$ saline (as control), CD-PLLD/DOC, CD-PLLD/MMP-9 or CD-PLLD/DOC/MMP-9, using DOC doses of $10 \mu \mathrm{g} / \mathrm{g}$ and MMP-9 doses of $3 \mu \mathrm{g} / \mathrm{g}$ according to the loading amount of DOC $(13.20 \mu \mathrm{g} / \mathrm{mg})$ and CD-PLLD/MMP-9 at the N/P ratios of 20 (16), on days 1, 6, 11 and 16, respectively. After three weeks, all mice were sacrificed by cervical dislocation. The tumor volume was calculated by the following formula: widest diameter $\mathrm{x}$ longest diameter ${ }^{2} / 2$. The tumors were dissected and fixed in $10 \%$ neutral buffered formalin, embedded in paraffin and sectioned at a thickness of $5 \mu \mathrm{m}$. The proliferating cell nuclear antigen (PCNA) was used to evaluate proliferation ability of the cells in the HNE-1 tumors. The PCNA expression in sections was assayed via immunohistochemistry with the Elivision two-step detection kit (Kit-0015; Fuzhou Maixin Biotech. Co., Ltd., China). These sections were deparaffinized and pretreated by boiling the slides in citrate buffer ( $\mathrm{pH} \mathrm{6.0)}$ for $10 \mathrm{~min}$. The sections were then immersed in $3 \%$ hydrogen peroxide for $10 \mathrm{~min}$ at room temperature to block endogenous tissue peroxidase activity. Following washing with phosphate buffered saline (PBS), sections were incubated with mouse PCNA monoclonal antibody (catalog no. TA800876; 1:50; Origene Technologies, Beijing, China) at $4^{\circ} \mathrm{C}$ overnight, and then sections were incubated with $50 \mu \mathrm{l}$ amplifier polymer (reagent $\mathrm{A}$ ), and $50 \mu \mathrm{l}$ horse radish peroxidase-conjugated anti-mouse/rabbit IgG (reagent B, Kit-0015; Fuzhou Maixin Biotech. Co., Ltd.) for $30 \mathrm{~min}$ at room temperature. Following washing with PBS, the sections were stained with DAB (3,3-dimethylbenzidine; Fuzhou Maixin Biotech. Co., Ltd.) and counterstained with hematoxylin (Fuzhou Maixin Biotech. Co., Ltd.), differentiated using hydrochloric acid in ethanol, blued by washing with water and sealed with conventional resin. The PCNA expression was examined with tan or brown staining in cell nucleus and no specific color in cytoplasm by light microscopy. The PCNA positive rates as positive cell index $(\mathrm{PI})$ were calculated in five visual fields $(\mathrm{x} 400)$ selected randomly in each section.

Statistical analysis. The peak area, DOC concentration and tumor size in each group were compared by two-way analysis of variance (ANOVA) and the PCNA expression of tumors in each group were compared by one-way ANOVA, followed by Tukey's post hoc test. Data was analyzed using SPSS software version 11.5 (SPSS, Inc., Chicago, IL, USA). All data are presented as the mean \pm standard deviation. $\mathrm{P}<0.05$ was considered to indicate a statically significant difference.

\section{Results}

In vivo metabolism assay. A linear association was determined between DOC concentration (X) and peak area (Y) in each tissue. The standard curve of DOC and regression equations were as follows: HNE-1 tumor, $\mathrm{Y}=2474.3 \mathrm{X}+799.96$; liver, $\mathrm{Y}=2693.0 \mathrm{X}-846.12$; kidney, $\mathrm{Y}=2655.5 \mathrm{X}+1238.8$; lung, $\mathrm{Y}=2581.3 \mathrm{X}-172.32$; heart, $\mathrm{Y}=2608.7 \mathrm{X}-701.39$; spleen, $\mathrm{Y}=2470.4$ $X+266.92$; and brain, $Y=2755.0 X+1459.2$. Following this, tissue DOC concentrations were calculated 5, 30 and $60 \mathrm{~min}$ after intravenous injection with CD-PLLD/DOC/MMP-9 complexes. HPLC analysis and DOC concentration in tissues

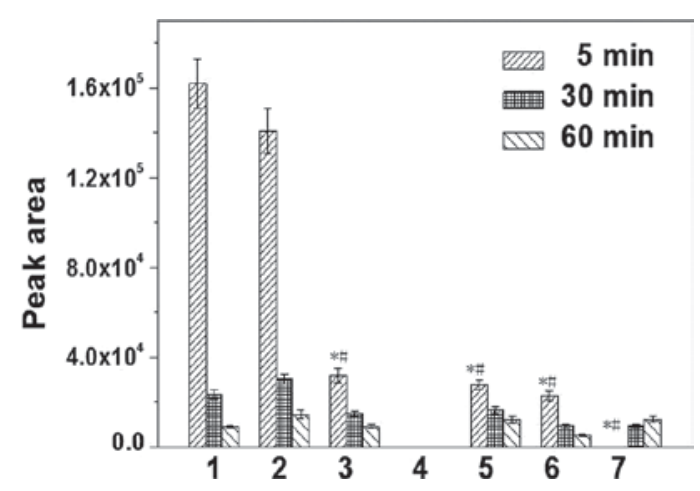

Figure 1. HPLC analysis of DOC in mouse tissues. BALB/c nude mice were implanted with HNE-1 human nasopharyngeal carcinoma cell tumors and intravenously injected with $\beta$-cyclodextrin-poly(L-lysine) dendron/DOC/matrix metalloproteinase 9. Tissues were harvested 5, 30 or 60 min later for HPLC analysis of DOC concentrations. Data are expressed as the mean \pm standard deviation. 1, liver; 2, kidney; 3, heart; 4, brain; 5, spleen; 6, lung; 7, HNE-1 tumor. ${ }^{*} \mathrm{P}<0.05$ vs. liver at $5 \mathrm{~min} ;{ }^{\#} \mathrm{P}<0.05$ vs. kidney at $5 \mathrm{~min}$. HPLC, high-performance liquid chromatography; DOC, docetaxel.

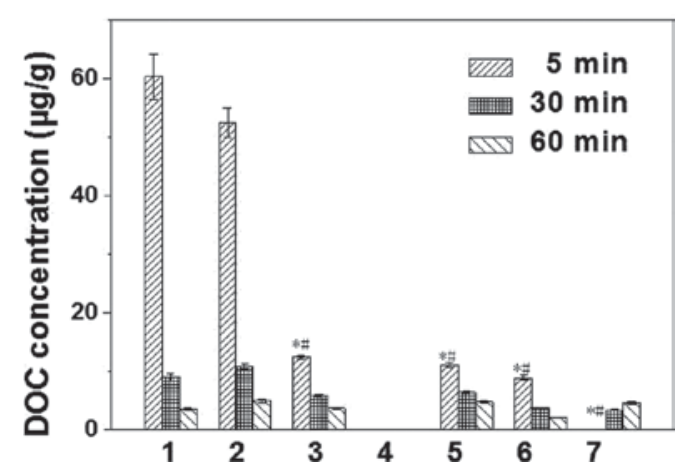

Figure 2. DOC concentrations in mouse tissues. BALB/c nude mice were implanted with HNE-1 human nasopharyngeal carcinoma cell tumors and intravenously injected with $\beta$-cyclodextrin-poly(L-lysine) dendron/DOC/matrix metalloproteinase 9 . Tissues were harvested 5, 30 or $60 \mathrm{~min}$ later for high performance liquid chromatography analysis of DOC concentrations. Data are expressed as the mean \pm standard deviation. 1, liver; 2, kidney; 3, heart; 4, brain; 5, spleen; 6, lung; 7, HNE-1 tumor. ${ }^{*} \mathrm{P}<0.05$ vs. liver at $5 \mathrm{~min} ;{ }^{~} \mathrm{P}<0.05 \mathrm{vs}$. kidney at $5 \mathrm{~min}$. DOC, docetaxel.

are presented in Figs. 1 and 2, respectively. The results demonstrated that DOC was absorbed rapidly and distributed widely in the majority of tissues, and that DOC concentration was the greatest in the liver and kidney, followed by the heart, spleen and lung $(\mathrm{P}<0.05)$. After $60 \mathrm{~min}$, DOC concentration markedly decreased, and there were no significant differences between organs $(\mathrm{P}>0.05)$. No DOC was detected in the brains of the nude mice. DOC concentrations in HNE-1 tumors increased with prolonged blood circulation. Following intravenous injection with CD-PLLD/DOC/MMP-9 complexes, EGFP expression after $48 \mathrm{~h}$ in frozen tissue sections is presented in Fig. 3. Similar to DOC distribution, EGFP expression was detectable in all assayed organs except the brain, and the liver and kidney frozen sections exhibited greater GFP expression. EGFP expression was observed in HNE-1 tumors, consistent with the DOC concentration analysis.

In vivo tumor therapy. The antitumor effects of CDPLLD/DOC/MMP-9 were assessed in vivo, using nude mice implanted with HNE-1 tumors. Representative 


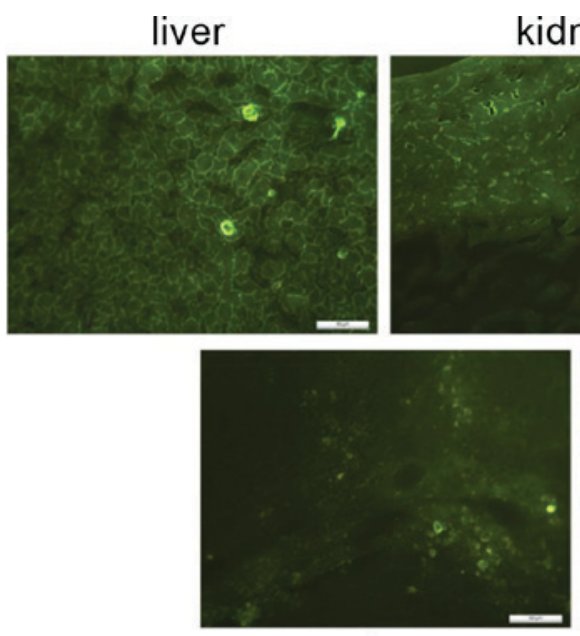

spleen kidney
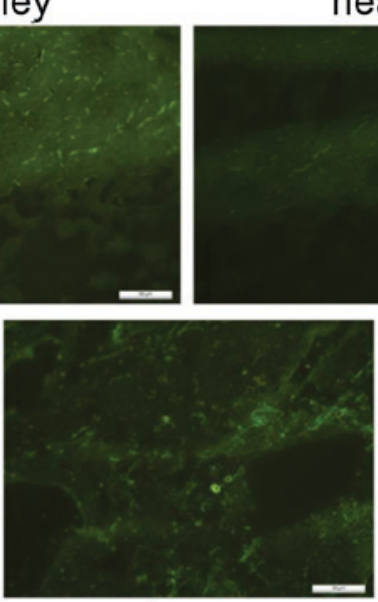

lung
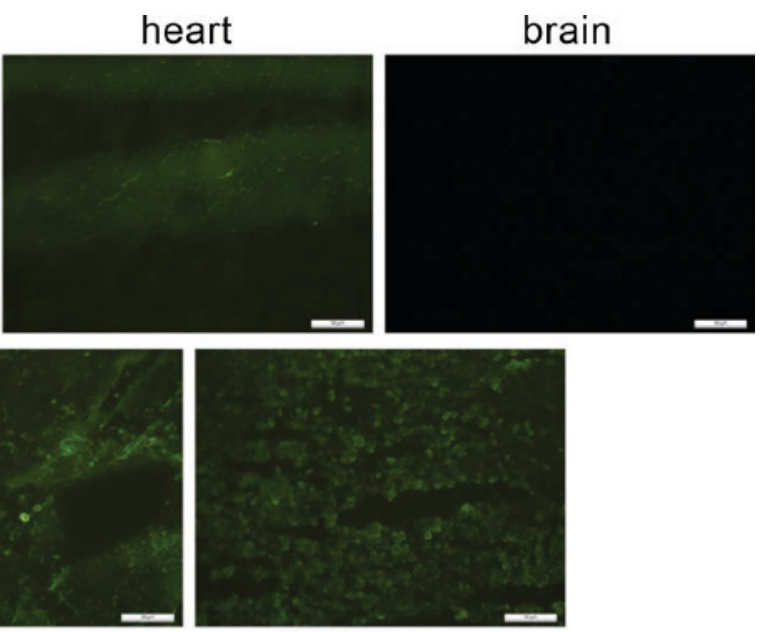

HNE-1 tumor

Figure 3. Expression of EGFP in mouse tissues. BALB/c nude mice were implanted with HNE-1 human nasopharyngeal carcinoma cell tumors and intravenously injected with $\beta$-cyclodextrin-poly(L-lysine) dendron/DOC/matrix metalloproteinase 9 . Tissues were harvested $48 \mathrm{~h}$ later for detection of GFP, a component of the complex. Original magnification, x200. GFP, green fluorescent protein.

A

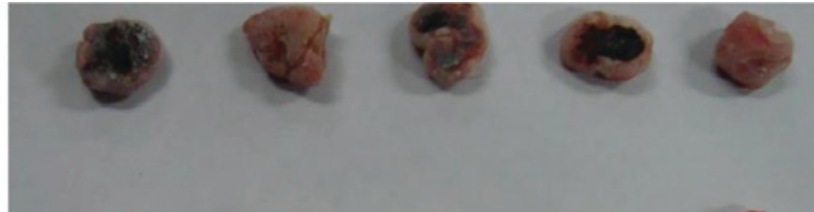

B

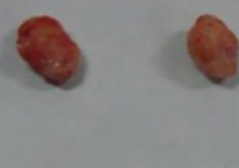

C

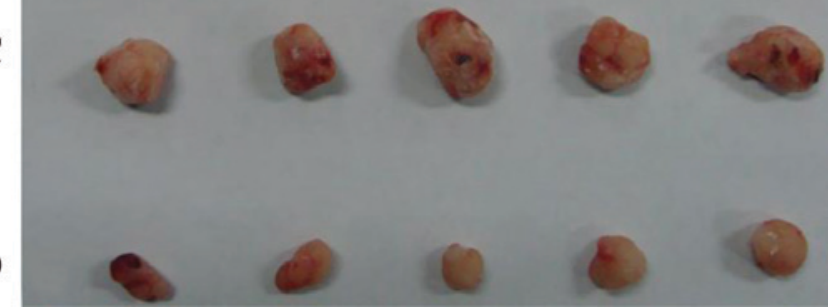

Figure 4. Tumor size following CD-PLLD/DOC/MMP-9 treatment. BALB/c nude mice were implanted with HNE-1 human nasopharyngeal carcinoma cell tumors and intravenously injected with saline, CD-PLLD/DOC, CD-PLLD/MMP-9 or CD-PLLD/DOC/MMP-9. Tumors were harvested 21 days later, and representative images are presented. (A) Saline control, (B)CD-PLLD/DOC,(C)CD-PLLD/MMP-9and(D)CD-PLLD/DOC/MMP-9 groups. CD-PLLD, $\beta$-cyclodextrin-poly(L-lysine) dendron; DOC, docetaxel MMP-9, matrix metalloproteinase 9.

tumor images are presented in Fig. 4. Tumor volume was measured every 3 days following implantation, until mice were sacrificed at day 21 (Fig. 5). Tumor volume in the saline-treated control group increased greatly during this period. Treatment with CD-PLLD/DOC, CO-PLLD/MMP-9 or CD-PLLD/DOC/MMP-9 inhibited tumor growth; CD-PLLD/DOC/MMP-9 to the greatest extent $(\mathrm{P}<0.05)$. Inhibition of tumor volume and weight were most marked in the CD-PLLD/DOC/MMP-9-treated group compared with the control group, with reductions of 61.32 and $60.00 \%$, respectively. Tumor volume and weight in the CD-PLLD/MMP-9-treated group were reduced 19.94 and $18.75 \%$, respectively, and in the CD-PLLD/DOC/MMP-9-treated group were reduced 41.40

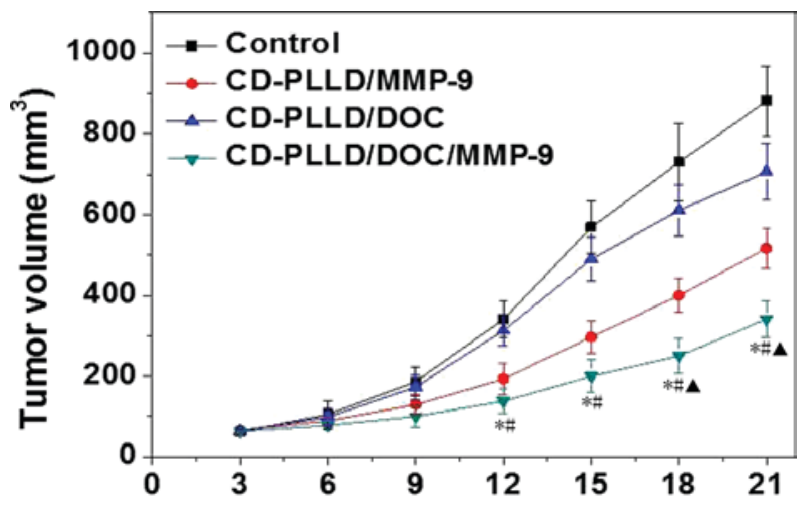

Figure 5. Tumor growth curve following CD-PLLD/DOC/MMP-9 treatment. BALB/c nude mice were implanted with HNE-1 human nasopharyngeal carcinoma cell tumors and intravenously injected with saline, CD-PLLD/DOC, CD-PLLD/MMP-9 or CD-PLLD/DOC/MMP-9. Tumor volume was monitored for 21 days. Data are expressed as the mean \pm standard deviation. ${ }^{*} \mathrm{P}<0.05$ vs. control group; ${ }^{\prime} \mathrm{P}<0.05$ vs. CD-PLLD/MMP-9 group; ${ }^{\wedge} \mathrm{P}<0.05$ vs. CD-PLLD/DOC group. CD-PLLD, $\beta$-cyclodextrin-poly (L-lysine) dendron; DOC, docetaxel; MMP-9, matrix metalloproteinase 9.

and $40.00 \%$, respectively, compared with the control group. Tumor PCNA expression in each group is presented in Fig. 6. Compared with the control group, PCNA expression levels in the CD-PLLD/MMP-9 group were slightly reduced, with a positive cell index (PI) of 80.62 vs. $92.83 \%$ in the control group; however, no significant differences were observed $(\mathrm{P}>0.05)$. PI in the CD-PLLD/DOC and CD-PLLD/DOC/MMP-9 groups were markedly reduced compared with the control group (65.36 and $39.52 \%$, respectively), to a greater extent in the CD-PLLD/DOC/MMP-9 group $(\mathrm{P}<0.05)$.

\section{Discussion}

NPC is one of the most common types of malignant tumor in southern China. Currently, a combination of radiotherapy and chemotherapy is the primary clinical treatment for NPC. However, the average five-year survival rate of advanced NPC patients remains $\sim 50 \%$ due to tumor metastasis and multi-drug 
A

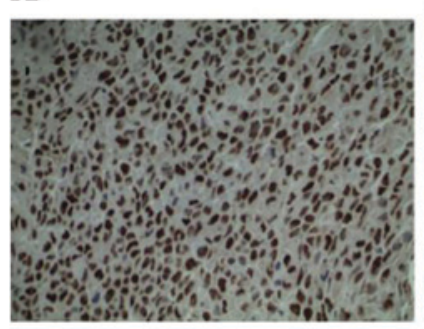

B

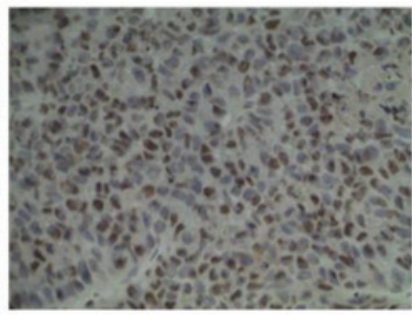

C

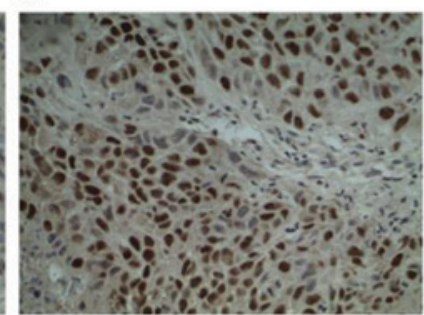

D

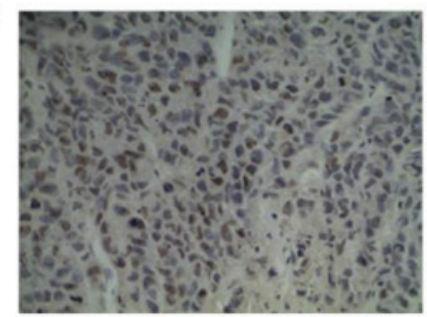

E

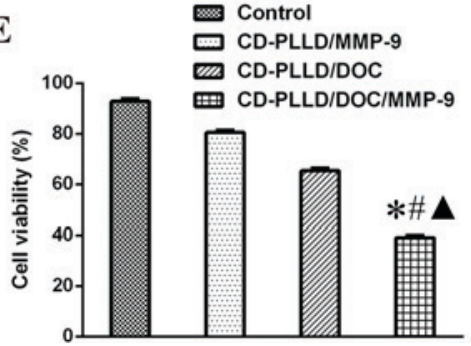

Figure 6. Expression of PCNA in tumors following CD-PLLD/DOC/MMP-9 treatment. BALB/c nude mice were implanted with HNE-1 human nasopharyngeal carcinoma cell tumors and intravenously injected with saline, CD-PLLD/DOC, CD-PLLD/MMP-9 or CD-PLLD/DOC/MMP-9. Tumors were harvested 21 days later and sections were stained for PCNA. (A) Saline control, (B) CD-PLLD/DOC, (C) CD-PLLD/MMP-9 and (D) CD-PLLD/DOC/MMP-9 groups. Original magnification, $\mathrm{x} 400$. (E) ${ }^{*} \mathrm{P}<0.05$ vs. control group; ${ }^{*} \mathrm{P}<0.05$ vs. CD-PLLD/MMP-9 group; ${ }^{\mathbf{A}} \mathrm{P}<0.05$ vs. CD-PLLD/DOC group. CD-PLLD, $\beta$-cyclodextrin-poly(L-lysine) dendron; DOC, docetaxel; MMP-9, matrix metalloproteinase 9; PCNA, proliferating cell nuclear antigen.

resistance. The co-delivery of drugs and genes has become a primary strategy for the treatment of cancer and other diseases, due to its potential to mediate synergistic actions, improve target selectivity and inhibit the development of drug resistance (17).

MMP-9, the enzyme with the largest molecular weight in the MMP family, may accelerate tumor invasion and metastasis due to its ability to degrade the basement membrane and extracellular matrix (18). It has previously been revealed to mediate tumor cell proliferation and inhibit apoptosis; therefore, MMP-9 inhibition via an siRNA plasmid may be a potential therapeutic strategy (19).

DOC, an analog of paclitaxel, is a novel anticancer agent of the taxoid family. It has been demonstrated to mediate tubulin assembly in microtubules and inhibit their depolymerization, leading to mitotic arrest in the $\mathrm{G}_{2} \mathrm{M}$ phase of the cell cycle (20,21). DOC may induce apoptosis of tumor cells and inhibit tumor growth, and has demonstrated antitumor effects in cancer chemotherapy $(22,23)$. However, DOC is poorly soluble in organic solvents and water and therefore does not disperse well in plasma. Taxotere ${ }^{\circledR}$, the clinical formulation of DOC, is formulated in polysorbate 80 , which has been identified to cause serious side-effects including hypersensitivity reactions, nephrotoxicity, cardiotoxicity and neurotoxicity (24), limiting the clinical application of DOC. Furthermore, single-drug chemotherapy may cause additional side-effects and tumor drug-resistance. Therefore, nanocarriers that co-deliver DOC and a target gene may provide a platform to overcome these limitations.

$\mathrm{CD}$ is a cyclic oligosaccharide with seven glucose units bonded by a $\alpha-1,4-$ linkage. It increases the water solubility of certain medicinal agents, via noncovalent inclusion complexation with a hydrophilic outer surface and a lipophilic central cavity, which encapsulates hydrophobic drug molecules or parts of these molecules. To co-deliver a chemotherapy drug and gene, our previous study prepared a biodegradable star-shaped CD-PLLD via chemical reactions with a $\mathrm{CD}$ surface modified with dendritic poly(L-lysine). The CD core interacted with the hydrophobic drug and the cationic arms bound to the target gene. It was demonstrated that such a conjugate may be used directly for drug and gene delivery without a complicated micellization process. In vitro assays indicated that CD-PLLD/DOC/MMP-9 complexes induce synergistic anti-tumor effects (16).

The present study investigated the in vivo applications for NPC therapy. Using a DOC distribution analysis, compared with the liquid-liquid anhydrous diethyl ether extraction method (25), it was demonstrated that tissue samples extracted by low-toxicity tert-butyl methyl ether are easily dried by nitrogen, acquiring an increased DOC extraction rate (26). Due to its size, the nanomedicine is easily delivered to the tumor cell, and accumulates in tumor tissues for enhanced permeability and retention $(27,28)$. Therefore, DOC concentrations in HNE-1 tumors increased with prolonged blood circulation. DOC concentrations were increased in organs rich in blood supply, including the liver and spleen, and decreased over time. GFP expression in each tissue was consistent with DOC distribution in vivo. These results indicated that CD-PLLD/DOC/MMP-9 complexes may be stable in the blood circulation. However, the complexes were easily phagocytized in reticuloendothelial systems including the liver and spleen; this requires further study. Furthermore, the complexes did not cross the blood-brain barrier due to their large molecular size, demonstrating their relative safety. Additionally, the complexes mediated increased DOC concentrations compared with prolonged blood circulation and EGFP expression in HNE-1 tumors.

In the present study, in a tumor-bearing mouse model, no significant differences were observed following treatment with CD-PLLD/MMP-9 compared with the control group. This may be due to removal of CD-PLLD/MMP-9 complexes by reticular endothelial cells in the liver and spleen, low gene 
concentrations in tumor, or because multiple genes are involved in tumor development, and therefore single-gene therapy is not effective. The antitumor effects were most marked in the CD-PLLD/DOC/MMP-9-treated group, as demonstrated by a clear decrease in PCNA expression levels. This may be due to the nano-characteristics of CD-PLLD, enabling it to accumulate DOC and MMP-9 in tumor tissue, and penetrate into the tumor to exert its effects in vivo (29). PCNA is a cofactor of DNA polymerase $\delta$, which is required for cell proliferation, and is therefore often used as an index of DNA replication and cell proliferation (30). The present study demonstrated that tumor PCNA expression levels in CD-PLLD/DOC/MMP-9-treated mice were significantly decreased and exhibited the greatest anti-tumor effects, as demonstrated by reduced weight and volume.

In conclusion, the present study investigated the co-delivery of a hydrophobic drug and target gene. In vivo assays demonstrated that CD-PLLD/DOC/MMP-9 may inhibit HNE-1 tumor growth and decrease PCNA expression levels. These results indicated a potential novel strategy for NPC therapy.

\section{Acknowledgements}

The present study was supported by the National Natural Science Foundation of China (grant nos. 81573000, 81260406, 81272687 and 81372477), the Guangdong Provincial Natural Science Foundation of China (grant no. 2015A030313864) and the Zhejiang Provincial Natural Science Foundation of China (grant no. LZ13H160004).

\section{References}

1. Wei WI and Sham JS: Nasopharyngeal carcinoma. Lancet 365 2041-2054, 2005.

2. Chan AT, Hsu MM, Goh BC, Hui EP, Liu TW, Millward MJ, Hong RL, Whang-Peng J, Ma BB, To KF, et al: Multicenter, phase II study of cetuximab in combination with carboplatin in patients with recurrent or metastatic nasopharyngeal carcinoma. J Clin Oncol 23: 3568-3576, 2005.

3. Dai $X$ and Tan C: Combination of microRNA therapeutics with small-molecule anticancer drugs: Mechanism of action and co-delivery nanocarriers. Adv Drug Deliv Rev 81: 184-197, 2015

4. He C, Liu D and Lin W: Self-assembled nanoscale coordination polymers carrying siRNAs and cisplatin for effective treatment of resistant ovarian cancer. Biomaterials 36: 124-133, 2015.

5. Li Y, Liu R, Yang J, Ma G, Zhang Z and Zhang X: Dual sensitive and temporally controlled camptothecin prodrug liposomes codelivery of siRNA for high efficiency tumor therapy. Biomaterials 35: 9731-9745, 2014.

6. Chang Y, Yang K, Wei P, Huang S, Pei Y, Zhao W and Pei Z: Cationic vesicles based on amphiphilic pillar [5] arene capped with ferrocenium: A redox-responsive system for drug/siRNA co-delivery. Angew Chem Int Ed Engl 53: 13126-13130, 2014.

7. Ma D, Zhang HB, Tu K and Zhang LM: Novel supramolecular hydrogel/micelle composite for co-delivery of anticancer drug and growth factor. Soft Matter 8: 3665-3672, 2012.

8. Wang AT, Liang DS, Liu YJ and Qi XR: Roles of ligand and TPGS of micelles in regulating internalization, penetration and accumulation against sensitive or resistant tumor and therapy for multidrug resistant tumors. Biomaterials 53: 160-172, 2015.

9. Hu Q, van Gaal EV, Brundel P, Ippel H, Hackeng T, Rijcken CJ, Storm G, Hennink WE and Prakash J: A novel approach for the intravenous delivery of leuprolide using core-cross-linked polymeric micelles. J Control Release 205: 98-108, 2015.

10. Ma D, Zhang HB, Chen YY, Lin JT and Zhang LM: New cyclodextrin derivative containing poly(L-lysine) dendrons for gene and drug co-delivery. J Colloid Interface Sci 405: 305-311, 2013.
11. Li M, Zhou X, Zeng X, Wang C, Xu J, Ma D and Xue W: Folate-targeting redox hyperbranched poly(amido amine)s delivering MMP-9 siRNA for cancer therapy. J Mater Chem B 4: 547-556, 2016.

12. Luo K, Li C, Li L, She W, Wang G and Gu Z: Arginine functionalized peptide dendrimers as potential gene delivery vehicles. Biomaterials 33: 4917-4927, 2012.

13. Ma D, Lin QM, Zhang LM, Liang YY and Xue W: A star-shaped porphyrin-arginine functionalized poly(L-lysine) copolymer for photo-enhanced drug and gene co-delivery. Biomaterials 35: 4357-4367, 2014

14. Pang JD, Zhuang BX, Mai K, Chen RF, Wang J and Zhang LM: Click modification of helical amylose by poly(l-lysine) dendrons for non-viral gene delivery. Mater Sci Eng C Mater Biol Appl 49: 485-492, 2015.

15. Ma D, Liu ZH, Zheng QQ, Zhou XY, Zhang Y, Shi YF, Lin JT and Xue W: Star-shaped polymer consisting of a porphyrin core and poly(L-lysine) dendron arms: Synthesis, drug delivery, and in vitro chemo/photodynamic therapy. Macromol Rapid Commun 34: 548-552, 2013.

16. Liu T, Xue W, Ke B, Xie MQ and Ma D: Star-shaped cyclodextrin-poly(L-lysine) derivative co-delivering docetaxel and MMP-9 siRNA plasmid in cancer therapy. Biomaterials 35: 3865-3872, 2014.

17. Creixell M and Peppas NA: Co-delivery of siRNA and therapeutic agents using nanocarriers to overcome cancer resistance. Nano Today 7: 367-379, 2012

18. Liotta LA, Steeg PS and Stetler-Stevenson WG: Cancer metastasis and angiogenesis: An imbalance of positive and negative regulation. Cell 64: 327-336, 1991.

19. Rao JS, Bhoopathi P, Chetty C, Gujrati M and Lakka SS: MMP-9 short interfering RNA induced senescence resulting in inhibition of medulloblastoma growth via p16(INK4a) and mitogen-activated protein kinase pathway. Cancer Res 67: 4956-4964, 2007.

20. Ajani JA: Chemotherapy for advanced gastric or gastroesophageal cancer: Defining the contributions of docetaxel. Expert Opin Pharmacother 7: 1627-1631, 2006.

21. Bouvier E, Thirot S, Schmidt F and Monneret C: First enzymatically activated Taxotere prodrugs designed for ADEPT and PMT. Bioorg Med Chem 12: 969-977, 2004.

22. Sánchez-Moreno P, Boulaiz H, Ortega-Vinuesa JL, Peula-García JM and Aránega A: Novel drug delivery system based on docetaxel-loaded nanocapsules as a therapeutic strategy against breast cancer cells. Int J Mol Sci 13: 4906-4919, 2012.

23. Cortes J, Rodriguez J, Aramendia JM, Salgado E, Gurpide A, Garcia-Foncillas J, Aristu JJ, Claver A, Bosch A, Lopez-Picazo JM, et al: Front-line paclitaxel/cisplatin based chemotherapy in brain metastases from non-small-cell lung cancer. Oncology 64: 28-35, 2003.

24. Feng SS and Chien S: Chemotherapeutic engineering: Application and further development of chemical engineering principles for chemotherapy of cancer and other diseases. Chem Eng Sci 58: 4087-4114, 2003

25. Zhao Y, Zhai D, Chen X, Yu Q, He H, Sun Y, Gao Z, Wang L, Wang H, Han D and Ji H: Determination of nimodipine in human plasma by HPLC-ESI-MS and its application to a bioequivalence study. J Chromatogr Sci 48: 81-85, 2010.

26. Ju P, Liu Z, Jiang Y, Zhao S, Zhang L, Zhang Y, Gu L, Tang X, Bi K and Chen X: Determination of a novel anticancer c-Met inhibitor LS-177 in rat plasma and tissues with a validated UPLC-MS/MS method: Application to pharmacokinetics and tissue distribution study. Biomed Chromatogr 29: 1103-1111, 2015.

27. Sledge GW Jr and Miller KD: Exploiting the hallmarks of cancer: The future conquest of breast cancer. Eur J Cancer 39: 1668-1675, 2003.

28. Teicher BA: Molecular targets and cancer therapeutics: Discovery, development and clinical validation. Drug Resist Updat 3: 67-73, 2000.

29. Brannon-Peppas L and Blanchette JO: Corrigendum to 'Nanoparticle and targeted systems for cancer therapy' Advanced Drug Delivery Reviews 56(2004)1649-1659. Adv Drug Deliv Rev 61: 364, 2009

30. Kato K, Kawashiri S, Yoshizawa K, Kitahara H, Okamune A, Sugiura S, Noguchi N and Yamamoto E: Expression form of p53 and PCNA at the invasive front in oral squamous cell carcinoma: Correlation with clinicopathological features and prognosis. J Oral Pathol Med 40: 693-698, 2011. 